\title{
Ultra-dense Hot Low Z Line Transition Opacity Simulations
}

Cite as: AIP Conference Proceedings 645, 352 (2002); https://doi.org/10.1063/1.1525475 Published Online: 15 November 2002

P. Sauvan, E. Mínguez, J. M. Gil, R. Rodríguez, J. G. Rubiano, P. Martel, P. Angelo, R. Schott, F. Philippe, E. LeboucherDalimier, R. Mancini, and A. Calisti

Export Citation

\section{Challenge us.}

What are your needs for periodic signal detection?

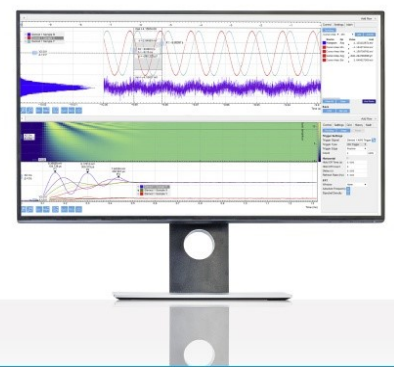

Zurich Instruments 


\title{
Ultra-dense Hot Low Z Line Transition Opacity Simulations
}

\author{
P.Sauvan*, E.Mínguez*, J.M.Gil ${ }^{\#}$, R.Rodríguez ${ }^{\#}$, J.G.Rubiano ${ }^{\#}$, P.Martel $^{\#}$, P.Angelo ${ }^{\ddagger}$, \\ R.Schott ${ }^{*}$, F.Philippe ${ }^{\ddagger}$, E. Leboucher-Dalimier ${ }^{*}$, R.Mancini ${ }^{\S}$, A.Calisti ${ }^{\ddagger}$ \\ *Instituto de Fusión Nuclear, C/ José Gutiérrez Abascal 2, 28006 Madrid, Spain

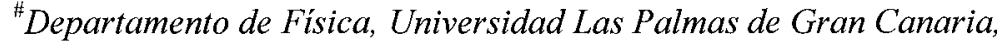 \\ 35017 Las Palmas de Gran Canaria, Spain \\ ${ }^{\ddagger}$ Physique Atomique dans les Plasmas Denses-LULI,CNRS-CEA-Ecole Polythecnique- \\ Université Paris 6, 75252 Paris cedex 05, France

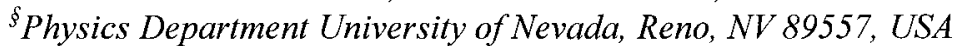 \\ "PIIM, Université de Provence, Centre St Jérome, 13397 Marseille, France
}

\begin{abstract}
In this work two atomic physics models (the IDEFIX code using the dicenter model and the code based on parametric potentials ANALOP) have been used to calculate the opacities for bound-bound transitions in hot ultra-dense, low $\mathrm{Z}$ plasmas. These simulations are in connection with experiments carried out at LULI during the last two years, focused on boundbound radiation. In this paper $\mathrm{H}$-like opacities for aluminum and fluorine plasmas have been simulated, using both theoretical models, in a wide range of densities and temperatures higher than $200 \mathrm{eV}$.
\end{abstract}

\section{INTRODUCTION}

The possibility to obtain fast and accurate opacity data for a wide range of plasma conditions is very useful when we have to study the radiation emitted from laser produced plasmas or astrophysical radiation. The use of analytical potentials reflecting the electronic structure of an ion immersed in the plasma is very useful to calculate in a very fast way the energy levels of this ion. Moreover, these potentials are appropriate for a large range of ionic configurations, that allows calculating the ionic energy levels in a lot of different plasmas conditions.

On the other hand, the dicenter model is able to calculate accurate ionic energies for plasmas at very high densities. In this work, we performed the first comparisons between the ANALOP code using the analytical potential and the IDEFIX code using the dicenter model. These comparisons are the preliminary works in order to extend the accuracy of the analytical potential results to the high-density range of plasmas.

An explanation of both models is being done in the next two sections, and a comparison focused on fluorine and aluminum Lyman $\beta$ lines provides the behavior of both codes.

CP645, Spectral Line Shapes: Volume 12, 16 $6^{\text {th }}$ ICSLS, edited by C. A. Back

(1) 2002 American Institute of Physics 0-7354-0100-4/02/\$19.00 


\section{THE DICENTER MODEL}

The relevance of the dicenter model to reproduce line shapes emitted from highly correlated plasmas has been demonstrated [1]. This model takes the advantage of treating exactly the interaction between the emitter and the first perturbing ion [2]. The numerical code IDEFIX, based on the dicenter approach, is able to calculate hydrogen and helium-like line shapes for high-density plasmas regime [3]. Recently, this code has been improved in order to include the effect of the surrounding plasma on the dicenter cell [4] (Molecular Stark effect). For one bound electron the corresponding Hamiltonian is:

$$
H(R, F, \Omega)=\frac{p^{2}}{2}+\frac{Z_{e}}{r_{1}}+\frac{Z_{p}}{r_{2}}+V_{e}-\vec{d} \cdot \vec{F}
$$

The first part of the Hamiltonian is the interaction of the bound electron with the quasi-molecule embedded in a free electron gas. The second part $(-\vec{d} \cdot \vec{F}$ term) is the linear Stark interaction of the bound electron with the screened ionic microfield produced by all ions in the plasma except the emitter and perturber. This Hamiltonian depends on three parameters, which are the distance between the two ions of the dicenter $\mathrm{R}$, the magnitude of the field $\mathrm{F}$, and the direction of the field with respect to the molecular axis $\Omega$. These two last parameters are arising from the scalar product $\vec{d} \cdot \vec{F}$.

\section{Absorption Cross Section In The Dicenter Model}

Since the Hamiltonian depends on the three parameters $\mathrm{R}, \mathrm{F}, \Omega$, the resulting energies and oscillator strengths will depend on these parameters. For one set of parameters $\mathrm{R}, \mathrm{F}, \Omega$, the expression of the absorption cross section is:

$$
\sigma_{\omega}^{a b s}(R, F, \Omega)=2 \pi^{2} \alpha \sum_{\text {lower }} \rho_{l} \frac{2}{3 g_{l}} \sum_{\text {upper }} \Delta E_{u-l}\left|\vec{d}_{u-l}\right|^{2} \frac{1}{\pi} \frac{\Phi_{u-l}}{\left(\Delta E_{u-l}-\omega\right)^{2}+\Phi_{u-l}^{2}}
$$

where $\Phi_{\mathrm{u}-1}$ is the electronic broadening operator, $\Delta \mathrm{E}_{\mathrm{u}-1}$ and $\left|\vec{d}_{u-l}\right|$ the transition energy and the dipole moment respectively are depending on R,F, $\Omega$. $\rho_{\mathrm{l}}, \mathrm{g}_{\mathrm{l}}$ and $\alpha$ stand for the population of the lower level 1, the statistical weight and fine structure constant respectively.

Each transition involved in the cross section is represented by a normalized Lorentzian profile, multiplied by the dipolar moment of the transition. The final cross section is obtained by summing all the cross sections depending on the parameters $\mathrm{R}, \mathrm{F}, \Omega$ taking into account the probability to find a given set of parameters for given plasma conditions. 


$$
\sigma_{\omega}^{a b s}=\int \sigma_{\omega}^{a b s}(R, F, \Omega) d R d F d \Omega
$$

\section{ANALYTICAL POTENTIAL MODEL}

The advantage of the analytical potentials in the calculation of atomic properties for the study of hot plasmas with respect to self-consistent calculations is the considerable reduction in the computing time. This reduction becomes more important when we develop models that handle each configuration into the plasma.

We have developed an opacity code called ANALOP [5] to calculate optical properties for plasmas based on analytical potentials. For an ion having a nuclear charge $\mathrm{Z}$ and $\mathrm{N}$ bound electrons embedded in the plasma, it was proposed the following effective analytical potential $[6,7]$

$$
\begin{gathered}
U_{e f f}(r)=-\frac{1}{r}\left\{(N-1)(\Phi(r)-\eta(r))+[Z-N+(N-1) \eta(0)]^{-a r}+1\right\} \\
\eta(r)=\frac{1}{2} a \int_{0}^{\infty} e^{-a|s-r|} \Phi(s) d s .
\end{gathered}
$$

where

$a$ being the inverse of the Debye radius given by

$$
a=\left(\frac{\rho Z^{*}\left(Z^{*}+1\right)}{k T}\right)^{1 / 2}
$$

Here $\rho$ is the ion density and $\Phi(r)$ is a screening function given by [8]

$$
\Phi\left(a_{1}, a_{2}, a_{3}, r\right)=\left\{\begin{array}{l}
e^{-a_{1} r^{a_{3}}}, \text { for } N>11 \\
\left(1-a_{2} r\right) e^{-a_{1} r} \quad, \text { for } 8 \leq N \leq 11 \text { or } N=2,3 \\
e^{-a_{1} r}, \text { for } 4 \leq N \leq 7
\end{array}\right.
$$

with

$$
a_{k}=c_{1 k} Z^{4}+c_{2 k} Z^{3}+c_{3 k} Z^{2}+c_{4 k} Z+c_{5 k}
$$

The coefficients $c_{i k}$ were obtained for the ground state of isolated ions from He-like to U-like [9].

This non-isolated parametric potential (4) has been obtained by solving the Poisson equation, assuming the linearized Debye-Hückel approximation, and taking into account the reaction of the plasma-charge density to the optical electron [6].

For a given ionic stage and into the context of the Independent Particle Model (IPM), the energy levels and orbital functions for each level (bound and free spectrum) 
is obtained by solving the Dirac equation using the effective potential given by (4). Because total energies are necessary for obtaining the ionic populations, an expression proposed in the Density Functional Theory [10] is used in ANALOP.

The expression of the bound-bound photo-absorption cross section for a given line is as following (in $\mathrm{a}_{0}{ }^{2}, \mathrm{a}_{0}$ is the Bohr radius)

$$
\sigma_{\omega}^{a b s}=2 \pi^{2} \alpha \overline{\phi(\bar{\omega})} \sum_{l} \rho_{l} \sum_{u} f_{u-l}^{a b s}
$$

Here $\rho_{l}$ stands for the populations of the lower mono-electronic levels involved in the transition, $\overline{\phi(\omega)}$ is the normalized Stark profile of the considered line. These profiles are calculated with the Pim Pam Poum (PPP) code [11] using as input data the energy transitions and oscillator strengths given by the ANALOP code.

In the equation (9), $f_{u-l}^{a b s}$ is the absorption oscillator strength, which is given into the context of IPM (the quantities in the equation are expressed in atomic units)

$$
f_{u l}^{a b s}=\frac{2 \Delta E_{u-l}}{3 g_{l}}\left|\left\langle\psi_{u}|r| \psi_{l}\right\rangle\right|^{2}
$$

Here $\quad E_{u-l}$ is the transition energy, $\psi_{k}$ is the normalized wavefunction associated to the monoelectronic level $k$, and $g_{l}$ is the statistical weight of the initial level.

Finally, the absorption coefficients for the bound-bound transitions have been evaluated, both with ANALOP and IDEFIX codes, through the following expressions

$$
\kappa_{\omega}=N_{l} \sigma_{\omega}^{a b s}
$$

where $\sigma_{\omega}^{a b s}$ stands for the photo-excitation cross section, $N_{l}$ is the abundance of ions in the initial state of the transition which is calculated with M3R code [12] using ANALOP energies and oscillator strengths.

\section{RESULTS}

In figure 1 we have plotted the profiles from the Dicenter Model and PPP for fluorine Lyman $\beta$ for the macroscopic plasma parameters $\mathrm{Ne}=5.10^{22} \mathrm{~cm}^{-3}$ and $\mathrm{Te}=500 \mathrm{eV}$. This figure shows a good agreement between both profiles at low density, being the differences between both intensities due to the fine structure effect not included in IDEFIX. 


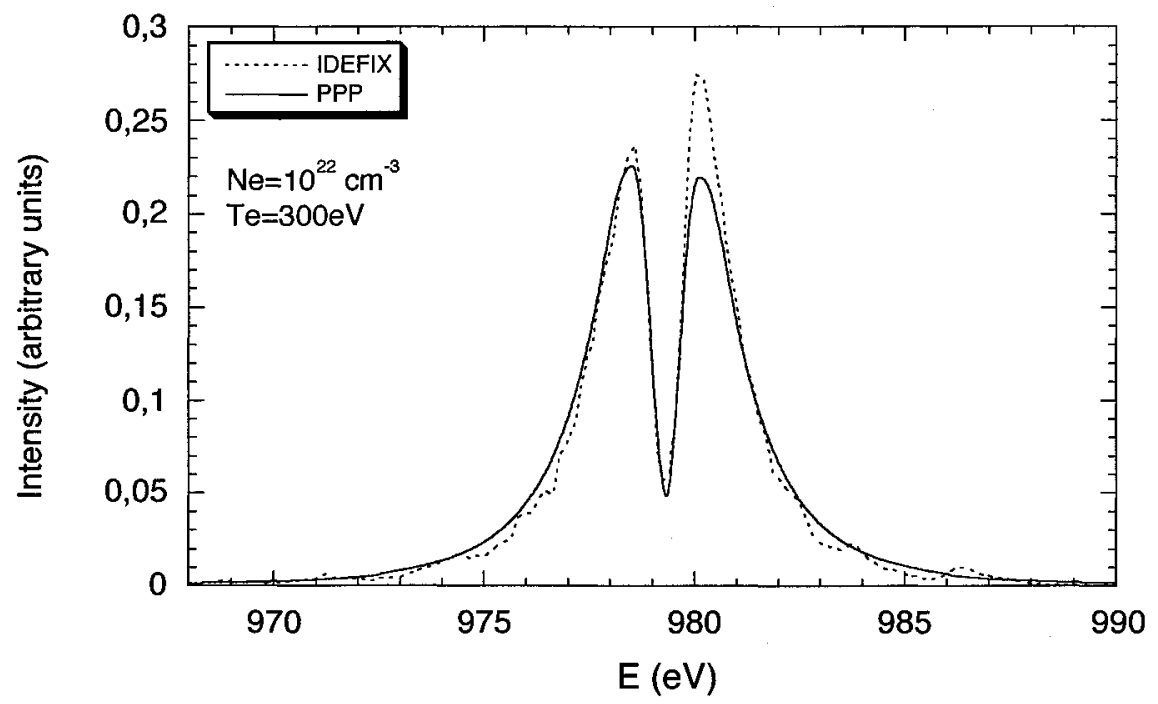

FIGURE 1. Profiles comparison for the fluorine Lyman $\beta$ between IDEFIX and PPP at "low" density.

However the divergence with the PPP profile increases rapidly with the density. Moreover, the Lyman $\beta$ line exhibits a stronger asymmetry as the density increases. These behaviors can be observed in figure 2 .

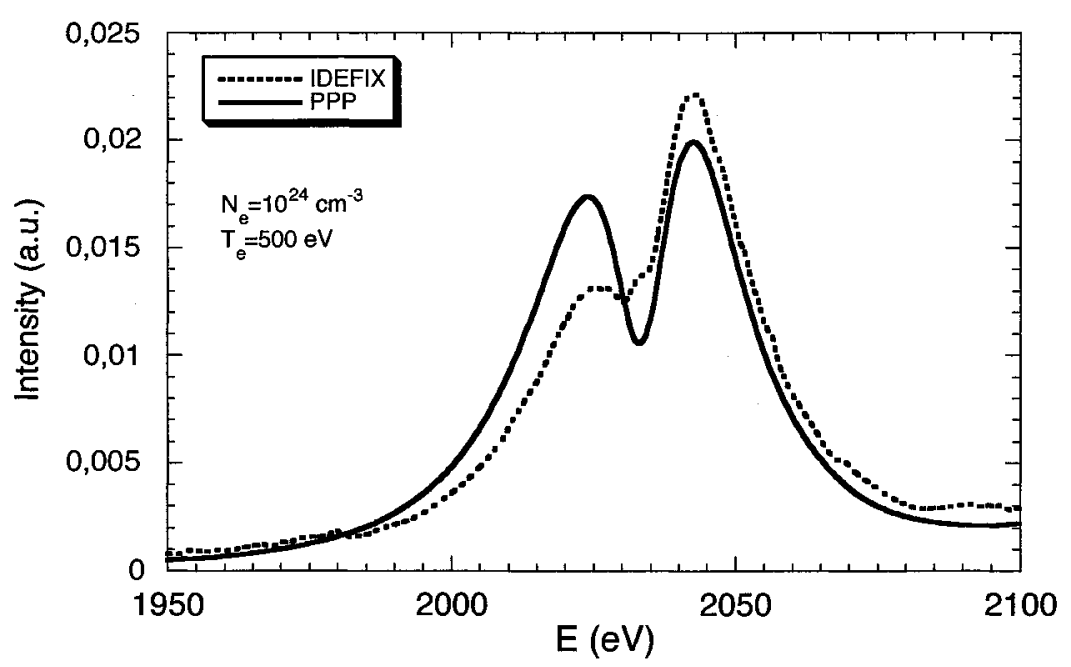

FIGURE 2. Profiles comparison for the aluminum Lyman $\beta$ between IDEFIX and PPP at high density.

In figure 3 we present the absorption cross section for aluminum Lyman $\beta$ for the macroscopic parameters $\mathrm{Ne}=5.10^{22} \mathrm{~cm}^{-3}$ and $\mathrm{Te}=500 \mathrm{eV}$. The plasma coupling 
parameter $\Gamma$, corresponding to these conditions is 1.1. It can be seen from the figure some discrepancies between the non-isolated analytical potential and the dicenter model. These discrepancies were expected, because of the use of the Debye-Hückel approximation in the determination of the non-isolated potential which is not valid at high densities, i.e. $\Gamma>1$. On the same figure we give results from the ion sphere potential model. They are close to IDEFIX results.

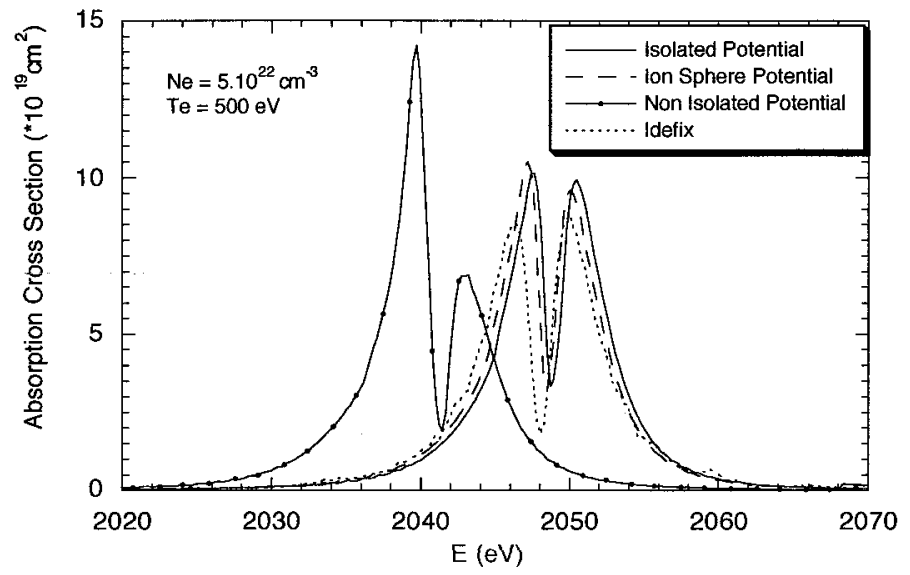

FIGURE 3. Absorption cross section for the aluminum Lyman $\beta . \Gamma=1.1$.

The analytical potential shows its applicability at low densities, i.e. with a coupling parameter less than one. In this range of density this potential reproduces the main effects of the plasma environment on the atomic magnitudes, such as the continuum lowering and the red shift of the lines with respect to the isolated situations, however, for low density the dicenter model is inapplicable. As an example in figure 4 the fluorine line profile at low density $(\Gamma=0.1)$ is shown.

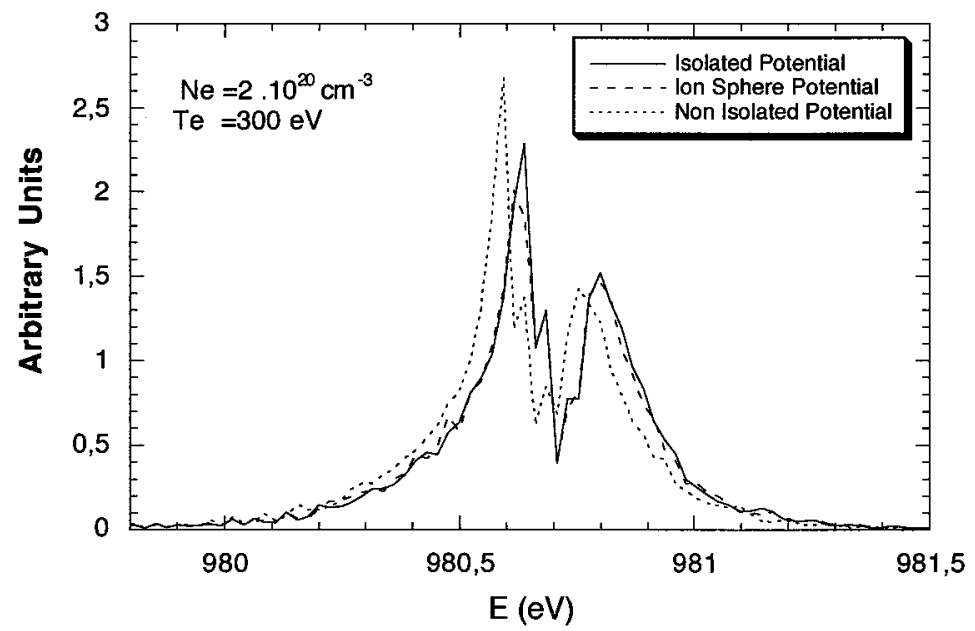

FIGURE 4. Fluorine Lyman $\beta$ line profile $(\Gamma=0.1)$. 


\section{CONCLUSIONS}

We have shown that the introduction of the plasma effect in the analytical potentials assuming the linearized Debye-Hückel approximation is valid at low density and as it was expected it gives very inaccurate results at high-density plasmas. At these high-densities, results with the ion sphere potential are closer to the dicenter model ones. A new non-isolated potential taking into account dense plasma effect is now under development which will give the non-isolated potential presented in this work for low densities, and the ion sphere model for highest densities.

\section{REFERENCES}

1. Leboucher-Dalimier, E., et al., J. Quant. Spectrosc. Radiat. Transfer 71, 493-504 (2001).

2. Gauthier, P., et al., Phys. Rev. E 58, 942-950 (1998).

3. Sauvan, P., et al., J Ouant. Spectrosc. Radiat. Transfer 65, 511-525 (2000).

4. Sauvan, P., et al., J. Quant. Spectrosc. Radiat. Transfer 71, 675-687 (2001).

5. Mínguez, E., et al., Nucl. Instrum. Methods A 415, 539 (1997).

6. Gil, J. M., et al., "Analytical Potentials Including Temperature and Density Effects for Calculation of Plasma Optical Properties" in Advances in Laser Interaction with Matter and Inerial Fusion, edited by Velarde G., Martínez-Val JM., Mínguez E. and Perlado JM., Pulisher World Scientific, Singapore 1997, pp. 385-388

7. Gil, J. M., et al., Submitted to J. Quant. Spectrosc. Radiat. Transfer (2002).

8. Martel, P., et al, J. Quant. Spectrosc. Radiat. Transfer 54, 621 (1995).

9. Martel, P., et al., J. Ouant. Spectrosc. Radiat. Transfer 4, 623 (1998).

10. Rajagopal, A. K., Adv. Chem. Phys. 41, 59 (1980).

11. Calisti, A., et al., Phys. Rev. A 42, 5433 (1990).

12. Mancini, R. C. and Minguez, E., First International NLTE Atomic Kinetic Workshop, Gaithersburg (1996) 